Mathematical Modelling And Analysis

Volume 19 Number 1, February 2014, 99-117

http://dx.doi.org/10.3846/13926292.2014.893455

(C) Vilnius Gediminas Technical University, 2014
Publisher: Taylor\&Francis and VGTU

http://www.tandfonline.com/TMMA

Print ISSN: 1392-6292

Online ISSN: 1648-3510

\title{
Numerical Solution of Higher Index Nonlinear Integral Algebraic Equations of Hessenberg Type Using Discontinuous Collocation Methods
}

\section{Babak Shiri}

Faculty of Mathematical Sciences, University of Tabriz

Tabriz, Iran

E-mail: shiri@tabrizu.ac.ir

Received January 29, 2013; revised January 8, 2014; published online February 20, 2014

\begin{abstract}
In this paper, we deal with a system of linear and nonlinear integral algebraic equations (IAEs) of Hessenberg type. Convergence analysis of the discontinuous collocation methods is investigated for the large class of IAEs based on the new definitions. Finally, some numerical experiments are provided to support the theoretical results.
\end{abstract}

Keywords: integral equation, collocation method, piecewise, integral algebraic equation, discontinuous piecewise collocation methods, continuous piecewise collocation methods.

AMS Subject Classification: 65R20; 45L05.

\section{Introduction}

Consider the system of integral equations of the form

$$
A(t) y(t)+\int_{0}^{t} k(t, s) y(s) d s=f(t), \quad t \in I:=[0, T]
$$

where matrix $A \in \mathbf{C}\left(I, R^{r \times r}\right)$, functions $f \in \mathbf{C}\left(I, R^{r}\right)$ and $k \in \mathbf{C}\left(D, R^{r \times r}\right)$ with $D:=\{(t, s): 0 \leq s \leq t \leq T\}$. If $A(t)$ is a singular matrix with constant rank for all $t \in I$, then the system (1.1) will be an IAE or a singular system of Volterra integral equations of the fourth kind.

The properties of IAEs are very similar to the differential algebraic equations (DAEs). Indeed, a simple integration of DAEs change them to IAEs. So the study of IAEs has important effect on the study of DAEs.

We recall the DAEs and IAEs classification by the index notions. There are many index notions by considering analytical and numerical properties of solutions $[2,5,6,7,8,10,12,13,18,19]$. In this paper, we introduce the "rankdegree" index and we use it for simplification of our analysis. 
We study the numerical properties of integral algebraic equations of Hessenberg type:

$$
\begin{aligned}
& {\left[\begin{array}{cccc}
A_{1,1}(t) & \cdots & A_{1, \nu-1}(t) & 0 \\
\vdots & \ddots & \vdots & \vdots \\
A_{\nu-1,1}(t) & \cdots & 0 & 0 \\
0 & \cdots & 0 & 0
\end{array}\right]\left[\begin{array}{c}
y_{1}(t) \\
y_{2}(t) \\
\vdots \\
y_{\nu}(t)
\end{array}\right]} \\
& +\int_{0}^{t}\left[\begin{array}{cccc}
k_{1,1}(t, s) & k_{1,2}(t, s) & \cdots & k_{1, \nu}(t, s) \\
\vdots & \vdots & \ddots & \vdots \\
k_{\nu-1,1}(t, s) & k_{\nu-1,2}(t, s) & \cdots & 0 \\
k_{\nu, 1}(t, s) & 0 & \cdots & 0
\end{array}\right]\left[\begin{array}{c}
y_{1}(s) \\
y_{2}(s) \\
\vdots \\
y_{\nu}(s)
\end{array}\right] d s=\left[\begin{array}{c}
f_{1}(t) \\
f_{2}(t) \\
\vdots \\
f_{\nu}(t)
\end{array}\right],
\end{aligned}
$$

where $\prod_{i=1}^{\nu-1} A_{i, \nu-i}$ and $\prod_{i=1}^{\nu} k_{i, \nu+1-i}(t, t)$ are assumed to be invertible and $A_{i, j}, k_{i, j}(t, t)$ and $f_{j}(t)$ are matrix functions of size $r_{i} \times r_{j}, r_{i} \times r_{j}$ and $r_{i} \times 1$ respectively. Let $r=r_{1}+\cdots+r_{\nu}$, with $r_{i}=r_{\nu+1-i}$ and $r_{i}=r_{\nu-i}, i=$ $1, \ldots, \nu-1$. Then $r_{1}=r_{2}=\cdots=r_{\nu}$. We use the letter $r$ for the size of system and the letter $r_{1}$ instead of $r_{i}$ for $i=1, \ldots, \nu$. For the sake of simplicity we consider the following first kind system of Volterra integral equations for $\nu=1$

$$
\int_{0}^{t} k_{1,1}(t, s) y_{1}(s) d s=f_{1}(t)
$$

and we suppose that $k_{1,1}$ is an invertible matrix function of size $r \times r$.

The piecewise polynomial collocation methods are popular methods for solving various types of operator equations, such as integral, differential and partial differential equations. From many existing papers on this subject, those which are close to our study are $[3,4,5,14,15,17]$. The piecewise discontinuous polynomial collocation methods for IAEs with differential index 1 of the form

$$
\begin{aligned}
y(t)+K_{11} y(t)+K_{12} z(t) & =q_{1}(t), \\
K_{21} y(t)+K_{22} z(t) & =q_{2}(t)
\end{aligned}
$$

have been investigated by Kauthen [16], where $K_{i, j} y(t)=\int_{0}^{t} k_{i j}(t, s) y(s) d s$ for $i, j \in\{1,2\}$ with det $k_{22} \neq 0$. This paper deals with application of discontinuous piecewise polynomial collocation method on higher index IAEs of Hessenberg form, since there is less investigation on these equations and their analyses are not as easy as the index one IAEs.

The next sections are organized as follows: In Section 2, we introduce a new definition based on the left index. In Section 3, we recall application of piecewise polynomial collocation method for the system (1.1). In Section 4, a global convergence theorem is proved which implies the convergent properties of the given methods for the research problem given in [5, p. 499]. In Section 5, we extend the results for nonlinear case. In Section 6 , we illustrate the results of paper by numerical experiments. Finally, in Section 7, we extend our results to the IAEs which are strongly equivalent to the IAEs of Hessenberg form. 


\section{Existence and Uniqueness of Solution}

Definition 1. [8] The matrix $A^{-}(t)$ is called semi-inverse matrix for $A(t)$ if it satisfies the equation $A(t) A^{-}(t) A(t)=A(t)$, which can be rewritten as $V(t) A(t)=0$, with $V(t)=\mathbf{I}-A(t) A^{-}(t)$, where $\mathbf{I}$ is an $r \times r$ identity matrix.

Definition 2. [20] Suppose $A \in \mathbf{C}\left(I, R^{r \times r}\right)$ and $k \in \mathbf{C}\left(D, R^{r \times r}\right)$. Let

$$
\begin{aligned}
& A_{0} \equiv A, \quad k_{0} \equiv k, \quad \Lambda_{i} y=\frac{d}{d t}\left(\left(\mathbf{I}-A_{i}(t) A_{i}^{-}(t)\right) y\right)+y \\
& A_{i+1} \equiv A_{i}+\left(\mathbf{I}-A_{i}(t) A_{i}^{-}(t)\right) k_{i}(t, t), \quad k_{i+1}=\Lambda_{i} k_{i}, \quad i=0, \ldots, \nu-1 .
\end{aligned}
$$

Then we say that the 'rank degree' index of $(A, k)$ is $\nu$ if

$$
\begin{aligned}
& k_{i}(t) \in \mathbf{C}^{1}\left(I, R^{r \times r}\right) \text { for } i=1, \ldots, \nu, \\
& \operatorname{rank} A_{i}(t)=\text { const., } \forall t \in I \text { for } i=0, \ldots, \nu, \\
& \operatorname{det} A_{i}=0, \quad \text { for } i=0, \ldots, \nu-1, \quad \operatorname{det} A_{\nu} \neq 0 .
\end{aligned}
$$

Moreover, we say that the 'rank-degree' index of system (1) is $\nu\left(i n d_{r}=\nu\right)$ if in addition to the above hypotheses, we have $f \in \mathbf{C}\left(I, R^{r}\right)$ and

$$
F_{0} \equiv f, \quad F_{i+1} \equiv \Lambda_{i} F_{i}, \quad i=0, \ldots, \nu-1, \quad F_{i} \in \mathbf{C}^{1}\left(I, R^{r}\right), \quad i=1, \ldots, \nu,
$$

where $\mathbf{I}$ is an identity operator.

Lemma 1. [9] Let $\operatorname{rank} A(t)=$ const., $\forall t \in I$ and the elements of $A(t)$ are in $\mathbf{C}^{p}\left(I, R^{r \times r}\right)$. If $\operatorname{rank}\left(\mathbf{I}-A A^{-}\right)=\operatorname{deg} \operatorname{det}\left(\lambda\left(\mathbf{I}-A A^{-}\right)+\left(\boldsymbol{I}-A A^{-}\right)^{\prime}+\mathbf{I}\right)=$ const., then the initial value problem

$$
\left(\left(\boldsymbol{I}-A A^{-}\right) x(t)\right)^{\prime}+x(t)=0, \quad x(0)=0
$$

has only trivial solution on $I$.

Now, we can state the following theorem of the uniqueness and existence for higher index IAEs.

Theorem 1. Suppose the following conditions are satisfied for (1.1):

1. $i n d_{r}=\nu \geq 1$,

2. $A_{i}(t) \in \mathbf{C}^{1}\left(I, R^{r \times r}\right), F_{i}(t) \in \mathbf{C}^{1}\left(I, R^{r}\right)$ and $k_{i} \in \mathbf{C}^{1}\left(\mathbb{D}, R^{r \times r}\right)$ for $i=$ $1, \ldots, \nu$,

3. $A_{i}(0) A_{\nu}^{-1}(0) F_{\nu}(0)=F_{i}(0)$ for $i=0, \ldots, \nu-1$ (consistency conditions),

4. $\operatorname{rank}\left(\mathbf{I}-A_{i} A_{i}^{-}\right)=\operatorname{deg} \operatorname{det}\left(\lambda\left(\mathbf{I}-A_{i} A_{i}^{-}\right)+\left(\boldsymbol{I}-A_{i} A_{i}^{-}\right)^{\prime}+\mathbf{I}\right)=$ const.

Then the system (1.1) has a unique solution on I. 
Proof. The proof stands on the fact that the systems

$$
A_{i}(t) y(t)+\int_{0}^{t} k_{i}(t, s) y(s) d s=F_{i}(t), \quad t \in I:=[0, T], i=0, \ldots, \nu
$$

are equivalent (i.e., every solution of the $(i+1)$ th system is a solution of $i$ th system and vice versa, if the consistency conditions are satisfied). To prove this assertion, let $y$ be a solution of $i$ th system and operate the operator $\Lambda_{i}$ on the $i$ th system. Then the $(i+1)$ th system will be obtained. Hence, $y$ is a solution of the $(i+1)$ th system. Conversely, let $y$ be a solution of $(i+1)$ th system. Define

$$
D_{i} x:=A_{i}(t) x(t)+\int_{0}^{t} k_{i}(t, s) x(s) d s-F_{i}(t)
$$

then

$$
\frac{d\left(\left(\mathbf{I}-A_{i}(t) A_{i}^{-}(t)\right) D_{i} y\right)}{d t}+D_{i} y=0, \quad D_{i} y(0)=A_{i}(0) y(0)-F(0)=0,
$$

since $y$ is a solution of $(i+1)$ th system and from Lemma 1 we have $D_{i} y=0$. Since the final system is a system of second kind Volterra integral equations, it has a unique solution. Therefore, a unique solution satisfies all systems in (2.1).

To prove existence of a solution for the system (1.2), it is enough to show its index is $\nu$, and the consistency conditions hold. The proof is simple and we omit it.

\section{Collocation Method for IAEs}

The content of this section is recalled from [5]. Let

$$
I_{h}:=\left\{t_{n}: 0=t_{0}<t_{1}<\cdots<t_{N}=T\right\}
$$

be a given (not necessarily uniform) partition of $I$, and set $\sigma_{n}:=\left(t_{n}, t_{n+1}\right]$, $\bar{\sigma}_{n}:=\left[t_{n}, t_{n+1}\right]$, with $h_{n}=t_{n+1}-t_{n}(n=0,1, \ldots, N-1)$ and diameter $h=$ $\max \left\{h_{n}: 0 \leq n \leq N\right\}$. Each component of the solution of (1.1) is approximated by elements of the discontinuous piecewise polynomial space

$$
\mathbb{S}_{m-1}^{(-1)}\left(I_{h}\right):=\left\{v:\left.v\right|_{\bar{\sigma}_{n}} \in \pi_{m-1}(n=0,1, \ldots, N-1)\right\},
$$

where $\pi_{m}$ denotes the space of all (real valued) polynomials of degree not exceeding $m$. A collocation solution $u_{h} \in\left(\mathbb{S}_{m-1}^{(-1)}\left(I_{h}\right)\right)^{r}$ for (1.1) is defined by the equation

$$
A(t) u_{h}(t)+\int_{0}^{t} k(t, s) u_{h}(s) d s=f(t)
$$

for $t \in X_{h}=\left\{t_{n, i}:=t_{n}+c_{i} h_{n}: 0<c_{1}<\ldots<c_{m} \leq 1, n=0, \ldots, N-1\right\}$. 
The collocation parameters $c_{i}$ completely determine the set of collocation points $X_{h}$. By defining $u_{n}=\left.u_{h}\right|_{\bar{\sigma}_{n}} \in\left(\pi_{m-1}\right)^{r}$, we have

$$
u_{n}\left(t_{n}+s h_{n}\right)=\sum_{j=1}^{m} L_{j}(s) U_{n, j}, \quad s \in(0,1], \quad U_{n, i}:=u\left(t_{n, i}\right),
$$

where the polynomials

$$
L_{j}(v):=\prod_{\substack{k=0 \\ k \neq j}}^{m} \frac{v-c_{k}}{c_{j}-c_{k}}, \quad j=0, \ldots, m
$$

denote the Lagrange fundamental polynomials with respect to the distinct collocation parameters $c_{i}$. By partitioning the domain of integral in (3.2) and changing of variable, we have

$$
A\left(t_{n, i}\right) U_{n, i}+F_{n, i}+h \int_{0}^{c_{i}} k\left(t_{n, i}, t_{n}+s h_{n}\right) u_{n}\left(t_{n}+s h_{n}\right) d s=f\left(t_{n, i}\right)
$$

where the lag terms are defined by

$$
F_{n, i}=h \sum_{l=0}^{n-1} \int_{0}^{1} k\left(t_{n, i}, t_{l}+s h_{l}\right) u_{l}\left(t_{l}+s h_{l}\right) d s .
$$

By substituting from (3.3) in (3.4), for $i=1, \ldots m$, we obtain the $r m \times r m$ system

$$
A\left(t_{n, i}\right) U_{n, i}+h \sum_{j=1}^{m} \int_{0}^{c_{i}} k\left(t_{n, i}, t_{n}+s h_{n}\right) L_{j}(s) d s U_{n, j}=-F_{n, i}+f\left(t_{n, i}\right)
$$

with

$$
F_{n, i}=h \sum_{l=0}^{n-1} \sum_{j=1}^{m} \int_{0}^{1} k\left(t_{n, i}, t_{l}+s h_{l}\right) L_{j}(s) d s U_{l, j} .
$$

By solving the system (3.5), the approximate solution of (1.1) is determined at the collocation points and $t_{n+1}$ by

$$
u_{n}\left(t_{n+1}\right)=\sum_{j=1}^{m} L_{j}(1) u_{n}\left(t_{n, j}\right)
$$

Remark 1. To apply this method, it is necessary to compute the integrals which appeared in (3.5) and (3.6). To do this, we apply the following quadrature rule by using the same collocation parameters $c_{i}, i=0, \ldots, m$, such that the order of the quadrature rule to be at least the same order of the method $\left(\mathcal{O}\left(h^{m}\right)\right)$,

$$
\begin{gathered}
\int_{0}^{c_{i}} k\left(t_{n, i}, t_{n}+s h_{n}\right) L_{j}(s) d s \simeq a_{i, j} k\left(t_{n, i}, t_{n}+c_{j} h_{n}\right), \\
\int_{0}^{1} k\left(t_{n, i}, t_{l}+s h_{l}\right) L_{j}(s) d s \simeq b_{j} k\left(t_{n, i}, t_{l}+c_{j} h_{l}\right)
\end{gathered}
$$

with $a_{i, j}=\int_{0}^{c_{i}} L_{j}(t) d t$ and $b_{j}=\int_{0}^{1} L_{j}(t) d t$. If all integrals are computed by quadrature rule then the method will be called fully discretised. 
Some existence and uniqueness conditions for the solution of continuous collocation methods can be found in [5].

\section{Convergence Analysis}

First we state the generalization of Gronwall inequality to use it in the proof of stated convergence analysis. Note that, we write $v=\mathcal{O}\left(h^{m}\right)$ whenever $\|v\|=\mathcal{O}\left(h^{m}\right)$. We use throughout the paper max norm, (i.e. $\left\|\left[v_{i j}(t)\right]\right\|=$ $\left.\max _{i, j} \max _{t \in I}\left|v_{i j}(t)\right|\right)$.

Lemma 2. Let $B_{j}(j \geq 0)$ be a uniformly bounded sequence of $v \times v$ matrices, $M=\operatorname{diag}\left(\lambda_{1}, \ldots, \lambda_{v}\right)$ and $\lambda=\max _{i}\left|\lambda_{i}\right|$. Let also $\left\{E_{n}\right\}$ be a set of vectors with $E_{0}=\mathcal{O}\left(h^{u_{1}}\right)$ and

$$
E_{n} \leq M E_{n-1}+h \sum_{l=0}^{n-1} B_{l} E_{l}+\mathcal{O}\left(h^{u_{2}}\right), \quad n=1, \ldots, N
$$

Then

$$
\begin{aligned}
& \lim _{\substack{N \rightarrow \infty \\
N h=\text { const. }}}\left\|E_{N}\right\|=\infty \quad \text { for } \lambda>1, \\
& \lim _{\substack{N \rightarrow \infty \\
N h=\text { const. }}}\left\|E_{N}\right\| \leq \mathcal{O}\left(h^{\min \left\{u_{1}, u_{2}\right\}}\right) \quad \text { for } \lambda_{i} \in[-1,1), \quad i=1, \ldots, v .
\end{aligned}
$$

Proof. Using (Gronwall inequality) [5], it is easy and we omit it.

Now, we can investigate the convergence properties of perturbed discontinuous collocation methods for system of first kind Volterra integral equations (SFVIE). The proof of Theorem 2 in this section is similar to the standard technique introduced by De Hoog [14,15], Brunner and Kauthen [3, 4, 5, 17]. However, for the convenience of the readers and self-dependency of the paper, we give the proofs of all theorems in details.

Consider the SFVIE

$$
\int_{0}^{t} k(t, s) y(s) d s=f(t)
$$

where $k(t, t)$ is an invertible $r \times r$ matrix for all $t \in I$. We analyze convergence properties of the perturbed discontinuous polynomial collocation method. For solving SFVIE, we perturb the corresponding system as

$$
h \sum_{j=1}^{m} \int_{0}^{c_{i}} k\left(t_{n, i}, t_{n}+s h_{n}\right) L_{j}(s) d s U_{n, j}=-F_{n, i}+f\left(t_{n, i}\right)+\delta(h, n, i),
$$

where

$$
F_{n, i}=h \sum_{l=0}^{n-1} \sum_{j=1}^{m} \int_{0}^{1} k\left(t_{n, i}, t_{l}+s h_{l}\right) L_{j}(s) d s U_{l, j},
$$

with $F_{0, i}=0$. Here, the perturbed term $\delta(h, n, i)$ depen ds only on $h$ and $t_{n, i}$, and it is of order $\mathcal{O}\left(h^{m_{1}}\right)$. 
Theorem 2. Let $f(t) \in \mathbf{C}^{m+1}\left(I, R^{r}\right), \frac{\partial^{i} k(t, s)}{\partial t^{i}} \in \mathbf{C}\left(D, R^{r \times r}\right)$ for $i=1, \ldots, m$ satisfy the system (4.2). Also suppose $k(t, t)$ be invertible matrix function for all $t \in I$, and $f(0)=0$. Then the approximate solution $u_{h}$ of the perturbed discontinuous collocation method with distinct collocation parameters $c_{1}, \ldots, c_{m} \in(0,1]$, and $c_{m} \leq 1$ (if exists) converges to the solution $y$ for any $m \geq 2$ if and only if

$$
-1 \leq \lambda=(-1)^{m} \prod_{i=1}^{m} \frac{1-c_{i}}{c_{i}} \leq 1
$$

and the collocation error satisfies

$$
\left\|y-u_{h}\right\| \leq \begin{cases}\mathcal{O}\left(h^{\min \left\{m_{1}-1, m\right\}}\right), & \text { if } \lambda \in[-1,1), \\ \mathcal{O}\left(h^{\min \left\{m_{1}-2, m-1\right\}}\right), & \text { if } \lambda=1\end{cases}
$$

Proof. Solving (4.3) and (4.4) is equivalent to find $u_{h}(s) \in\left(\mathbb{S}_{m-1}^{(-1)}\left(I_{h}\right)\right)^{r}$ such that

$$
\int_{0}^{t_{n i}} k\left(t_{n i}, s\right) u_{h}(s) d s=f\left(t_{n i}\right)+\delta(h, n, i) .
$$

Subtracting this equation from (4.2) with $t=t_{n, i}$, we obtain

$$
\int_{0}^{t_{n i}} k\left(t_{n i}, s\right) e(s) d s=\delta(h, n, i)
$$

where $e(s)=y(s)-u(s)$. Introducing $e_{n}=\left.e\right|_{\bar{\sigma}_{n}} \in \mathbf{C}^{m}\left[t_{n}, t_{n+1}\right]$, we have

$$
e_{n}\left(t_{n}+v h\right)=\sum_{j=1}^{m} L_{j}(v) e_{n}\left(t_{n, j}\right)+r_{n}(v), \quad v \in[0,1],
$$

where the interpolation error is determined by

$$
r_{n}(v)=h^{m} \frac{y^{(m)}\left(\xi_{n}(v)\right)}{(m) !} \prod_{i=1}^{m}\left(v-c_{i}\right), \quad \xi_{n}(v) \in\left(t_{n}, t_{n+1}\right) .
$$

(Notice that we have $f(t) \in \mathbf{C}^{m+1}\left(I, R^{r}\right)$ and $\frac{\partial^{i} k(t, s)}{\partial t^{i}} \in \mathbf{C}\left(D, R^{r \times r}\right)$ for $i=$ $1, \ldots, m$.) From $(4.6)$, we have

$$
\int_{0}^{c_{i}} k\left(t_{n i}, t_{n}+s h\right) e\left(t_{n}+s h\right) d s=-\sum_{l=0}^{n-1} \int_{0}^{1} k\left(t_{n i}, t_{l}+s h\right) e\left(t_{l}+s h\right) d s+\frac{\delta(h, n, i)}{h} .
$$

Rewriting (4.9) with $n$ replaced by $n-1$ and $i=m$, and subtracting it from (4.9), we obtain

$$
\begin{aligned}
\int_{0}^{c_{i}} k\left(t_{n i}, t_{n}+s h\right) e_{n}\left(t_{n}+s h\right) d s \\
\quad=\int_{0}^{c_{m}} k\left(t_{n-1, m}, t_{n-1}+s h\right) e_{n-1}\left(t_{n-1}+s h\right) d s
\end{aligned}
$$




$$
\begin{aligned}
& -\int_{0}^{1} k\left(t_{n i}, t_{n-1}+s h\right) e_{n-1}\left(t_{n-1}+s h\right) d s \\
& +\sum_{l=0}^{n-2} \int_{0}^{1}\left(k\left(t_{n-1, m}, t_{l}+s h\right)-k\left(t_{n i}, t_{l}+s h\right)\right) e_{l}\left(t_{l}+s h\right) d s \\
& +\frac{\delta(h, n, i)-\delta(h, n-1, m)}{h} .
\end{aligned}
$$

Using

$$
\begin{aligned}
\int_{0}^{c_{i}} k\left(t_{n i}, t_{n}+s h\right) L_{j}(s) d s & =k\left(t_{n}, t_{n}\right) a_{i j}+\mathcal{O}(h), \\
\int_{0}^{c_{m}} k\left(t_{n-1, m}, t_{n-1}+s h\right) L_{j}(s) d s & =k\left(t_{n}, t_{n}\right) a_{m j}+\mathcal{O}(h), \\
\int_{0}^{1} k\left(t_{n i}, t_{n-1}+s h\right) L_{j}(s) d s & =k\left(t_{n}, t_{n}\right) b_{j}+\mathcal{O}(h)
\end{aligned}
$$

and

$$
k\left(t_{n-1, m}, t_{l}+s h\right)-k\left(t_{n i}, t_{l}+s h\right)=h\left(1+c_{i}-c_{m}\right) k_{t}\left(\xi_{n}, t_{l}\right)+\mathcal{O}(h)
$$

in $(4.10)$, we obtain

$$
\begin{aligned}
& \left(k\left(t_{n}, t_{n}\right)+\mathcal{O}(h)\right)\left(\sum_{j=1}^{m} a_{i j} e_{n}\left(t_{n j}\right)+\mathcal{O}\left(h^{m}\right)\right) \\
& =\left(k\left(t_{n}, t_{n}\right)+\mathcal{O}(h)\right)\left(\sum_{j=1}^{m} a_{m j} e_{n-1}\left(t_{n-1, j}\right)+\mathcal{O}\left(h^{m}\right)\right) \\
& \quad-\left(k\left(t_{n}, t_{n}\right)+\mathcal{O}(h)\right)\left(\sum_{j=1}^{m} b_{j} e_{n-1}\left(t_{n-1, j}\right)+\mathcal{O}\left(h^{m}\right)\right) \\
& \quad+\left(h\left(1+c_{i}-c_{m}\right) k_{t}\left(\xi_{n}, t_{l}\right)+\mathcal{O}(h)\right) \sum_{l=0}^{n-2}\left(\sum_{j=1}^{m} b_{j} e_{l}\left(t_{l, j}\right)+\mathcal{O}\left(h^{m}\right)\right) \\
& +\frac{\delta(h, n, i)-\delta(h, n-1, m)}{h} .
\end{aligned}
$$

Since $k(t, t)$ is invertible and continuous with respect to $t, k\left(t_{n}, t_{n}\right)+\mathcal{O}(h)$ has continuous inverse, say $W$, for sufficiently small $h$. Hence, equation (4.11) can be written as

$$
\begin{aligned}
\sum_{j=1}^{m} a_{i j} e_{n}\left(t_{n j}\right)= & \sum_{j=1}^{m} a_{m j} e_{n-1}\left(t_{n-1, j}\right)-\sum_{j=1}^{m} b_{j} e_{n-1}\left(t_{n-1, j}\right) \\
& +h W U \sum_{l=0}^{n-2} \sum_{j=1}^{m} b_{j} e_{l}\left(t_{l, j}\right)+\mathcal{O}\left(h^{m_{1}-1}\right)+\mathcal{O}\left(h^{m}\right),
\end{aligned}
$$


where $h U:=h\left(1+c_{i}-c_{m}\right) k_{t}\left(\xi_{n}, t_{l}\right)+\mathcal{O}(h)$, and so $U$ is bounded with respect to its variables. Thus

$$
\begin{aligned}
\left(\begin{array}{ccc}
a_{11} \mathbf{I} & \cdots & a_{1 m} \mathbf{I} \\
\vdots & \ddots & \vdots \\
a_{m 1} \mathbf{I} & \cdots & a_{m m} \mathbf{I}
\end{array}\right)\left(\begin{array}{c}
e_{n}\left(t_{n, 1}\right) \\
\vdots \\
e_{n}\left(t_{n, m}\right)
\end{array}\right) \\
=\left(\begin{array}{ccc}
\left(a_{m 1}-b_{1}\right) \mathbf{I} & \cdots & \left(a_{m m}-b_{m}\right) \mathbf{I} \\
\vdots & \ddots & \vdots \\
\left(a_{m 1}-b_{1}\right) \mathbf{I} & \cdots & \left(a_{m m}-b_{m}\right) \mathbf{I}
\end{array}\right)\left(\begin{array}{c}
e_{n-1}\left(t_{n-1,1}\right) \\
\vdots \\
e_{n-1}\left(t_{n-1, m}\right)
\end{array}\right) \\
\quad+h \sum_{l=0}^{n-2} B_{l} E_{l}+\mathcal{O}\left(h^{m_{1}-1}\right)+\mathcal{O}\left(h^{m}\right),
\end{aligned}
$$

where $E_{l}=\left[e_{l}\left(t_{l, 1}\right), \ldots, e_{l}\left(t_{l, m}\right)\right]^{T}$ and $B_{l}$ are appropriate matrices. By using the Kronecker product, we summarize equation (4.13) in the form:

$$
(\widetilde{A} \otimes \mathbf{I}) E_{n}=(B \otimes \mathbf{I}) E_{n-1}+h \sum_{l=0}^{n-2} B_{l} E_{l}+\mathcal{O}\left(h^{u}\right), \quad u=\min \left\{m_{1}-1, m\right\} .
$$

Here $\widetilde{A}_{i j}=a_{i j}$ and $B_{i j}=\left(a_{m j}-b_{j}\right)$, for $i, j \in\{1, \ldots, m\}$. Since $\widetilde{A}$ and $\mathbf{I}$ are invertible matrices, we have

$$
E_{n}=\left(\left(\widetilde{A}^{-1} B\right) \otimes \mathbf{I}\right) E_{n-1}+h \sum_{l=0}^{n-2}\left(\widetilde{A}^{-1} \otimes \mathbf{I}\right) B_{l} E_{l}+\mathcal{O}\left(h^{u}\right) .
$$

From [5, Lemma 2.4.3], there exists an invertible matrix $P$ such that $D:=$ $P\left(\widetilde{A}^{-1} B\right) P^{-1}=\operatorname{diag}(\lambda, 0, \ldots, 0)$ with

$$
\lambda=(-1)^{m} \prod_{i=1}^{m} \frac{1-c_{i}}{c_{i}}
$$

Equation (4.15) can be written as

$$
\begin{aligned}
(P \otimes \mathbf{I}) E_{n}= & (D \otimes \mathbf{I})(P \otimes \mathbf{I}) E_{n-1} \\
& +h \sum_{l=0}^{n-2}(P \otimes \mathbf{I})^{-1}\left(\widetilde{A}^{-1} \otimes \mathbf{I}\right) B_{l}(P \otimes \mathbf{I})^{-1}(P \otimes \mathbf{I}) E_{l}+\mathcal{O}\left(h^{u}\right) .
\end{aligned}
$$

It is obvious that $E_{0}=\mathcal{O}\left(h^{\min \left\{m_{1}-1, m\right\}}\right)$. Thus we can use Lemma 2 to prove that

$$
\lim _{\substack{N \rightarrow \infty \\ N h=\text { const. }}}\left\|(P \otimes \mathbf{I}) E_{N}\right\|=\leq \mathcal{O}\left(h^{\min \left\{m_{1}-1, m\right\}}\right) .
$$

Then by using (4.7), the proof is completed for the case $\lambda \in[-1,1)$.

For the case $\lambda=1$, we use an indirect proof as follows, since it can not be proved directly. we apply the given ideas in [17] to use interpolation formula for $e(t)$ at the less number of collocation points, but this application in the perturbation case is not as simple as the ideas of [17] and we need the following remarks. 
Remark 2. Let $a_{i j}=\int_{0}^{c_{i}} \bar{L}_{j}(s) d s$ and $b_{j}=\int_{0}^{1} \bar{L}_{j}(s) d s$, where $\bar{L}_{j}(v), j=$ $1, \ldots, l$ are the Lagrange polynomials of degree $l$ defined at the points $0<$ $d_{1}<\cdots<d_{l}<1$. Then $M=A^{-1} B$ has the only eigenvalue

$$
\bar{R}(\infty)=(-1)^{l} \prod_{i=1}^{l} \frac{1-d_{i}}{d_{i}},
$$

where $A=\left(a_{i j}\right)$ and $B=\left(B_{i j}\right)$ with $B_{i j}=a_{m j}-b_{j}$. Moreover

$$
e\left(t_{n}+v h\right)=\sum_{j=1}^{l} \bar{L}_{j}(v) e_{n}\left(\bar{t}_{n, j}\right)+h^{m} \frac{e_{n}^{(l)}\left(\bar{t}_{n}^{+}\right)}{l !} \prod_{i=1}^{l}\left(\tau-c_{i}\right), \quad \tau \in[0,1],
$$

where $\bar{t}_{n, j}=t_{n}+d_{j} h$ (see for example [17]).

Remark 3. For the case $\lambda=1$ with respect to the collocation parameters $c_{1}, \ldots, c_{m}$, we know the matrix $M$ of Remark 2 has the only eigenvalue

$$
\bar{R}(\infty)=(-1)^{m} \prod_{i=1}^{m} \frac{1-c_{i}}{c_{i}}=1
$$

(see [17]). Hence, it can be easily proved that for each $l<m$, one can choose the $l$ points $d_{1}, \ldots, d_{l}$ through $c_{1}, \ldots, c_{m}$, such that

$$
\bar{R}(\infty)=(-1)^{l} \prod_{i=1}^{l} \frac{1-d_{i}}{d_{i}}<1 .
$$

Let $l_{1}=\min \{m 1-1, m\}$. Then, we use the above remarks with $l=l_{1}-1$ and the collocation points $d_{1}, \ldots, d_{l}$, to get

$$
e_{n}\left(t_{n}+v h\right)=\sum_{j=1}^{l} \bar{L}_{j}(v) e_{n}\left(t_{n, j}\right)+O\left(h^{l}\right), \quad v \in[0,1] .
$$

By substituting (4.18) into (4.10) and following the lines of proof after equation (4.10), we obtain

$$
E_{n}=(M \otimes \mathbf{I}) E_{n-1}+h \sum_{l=0}^{n-1} \overline{B_{n l}} E_{l}+O\left(h^{l}\right) .
$$

Since $M$ is diagonalizable, there exists an invertible matrix $P$ such that $D=$ $P M P^{-1}=\operatorname{diag}(\bar{R}(\infty), 0, \ldots, 0)$ with $|\bar{R}(\infty)|<1$. Now applying Lemma 2 to the equation

$$
(P \otimes \mathbf{I}) E_{n}=(D \otimes \mathbf{I})(P \otimes \mathbf{I}) E_{n-1}+h \sum_{l=0}^{n-1}(P \otimes \mathbf{I}) \overline{B_{n l}} E_{l}+O\left(h^{l}\right)
$$

completes the proof.

This perturbed analysis on the numerical solution of the system of first kind Volterra integral equations makes it easy for Hessenberg form of IAEs. 
Theorem 3. Let $f(t) \in \mathbf{C}^{m+1}\left(I, R^{r}\right), \frac{\partial^{i} k(t, s)}{\partial t^{i}} \in \mathbf{C}\left(D, R^{r \times r}\right)$ for $i=1, \ldots, m$ and $A(t) \in \mathbf{C}^{m}\left(I, R^{r \times r}\right)$ satisfy system (1.1). Suppose that this system has Hessenberg form of index $\nu$ (see Eq. (1.2)). Also suppose that the consistency conditions of Theorem 1 hold for this system. Then the approximate solution $u_{h}$ of the discontinuous collocation method with distinct collocation parameters $c_{1}, \ldots, c_{m} \in(0,1]$ (if exists) converges to the solution $y$ iff $-1 \leq \lambda \leq 1$. Then the collocation error satisfies

$$
\left\|y_{i}-u_{i}\right\| \leq \begin{cases}\mathcal{O}\left(h^{m+1-i}\right), & \text { if } \lambda \in[-1,1), \\ \mathcal{O}\left(h^{m+1-2 i}\right), & \text { if } \lambda=1\end{cases}
$$

for $i=1, \ldots, \nu$. Thus

$$
\left\|y-u_{h}\right\| \leq \begin{cases}\mathcal{O}\left(h^{m+1-\nu}\right), & \text { if } \lambda \in[-1,1), \\ \mathcal{O}\left(h^{m+1-2 \nu}\right), & \text { if } \lambda=1\end{cases}
$$

where $u_{h}=\left[u_{1}, \ldots, u_{\nu}\right]^{T}$.

Proof. The proof is done by induction on $\nu$. For the case $\nu=1$, it is trivial. Suppose that it is true for $\nu=n$. Let $\nu=n+1$, the problem takes the form

$$
\begin{aligned}
& {\left[\begin{array}{cccc}
A_{1,1}(t) & \cdots & A_{1, n}(t) & 0 \\
\vdots & \ddots & \vdots & \vdots \\
A_{n, 1}(t) & \cdots & 0 & 0 \\
0 & \cdots & 0 & 0
\end{array}\right]\left[\begin{array}{c}
y_{1}(t) \\
y_{2}(t) \\
\vdots \\
y_{n+1}(t)
\end{array}\right]} \\
& +\int_{0}^{t}\left[\begin{array}{cccc}
k_{1,1}(t, s) & k_{1,2}(t, s) & \cdots & k_{1, n+1}(t, s) \\
\vdots & \vdots & \ddots & \vdots \\
k_{n, 1}(t, s) & k_{k, 2}(t, s) & \cdots & 0 \\
k_{n+1,1}(t, s) & 0 & \cdots & 0
\end{array}\right]\left[\begin{array}{c}
y_{1}(t) \\
y_{2}(t) \\
\vdots \\
y_{n+1}(t)
\end{array}\right] d s=\left[\begin{array}{c}
f_{1}(t) \\
f_{2}(t) \\
\vdots \\
f_{n+1}(t)
\end{array}\right] .
\end{aligned}
$$

The last $n$ equations of this system form an IAE of index $n$. By the hypothesis of induction, we have

$$
\left\|e_{i}\right\| \leq \begin{cases}\mathcal{O}\left(h^{m+1-i}\right), & \text { if } \lambda \in[-1,1), \\ \mathcal{O}\left(h^{m+1-2 i}\right), & \text { if } \lambda=1\end{cases}
$$

for $i=1, \ldots, n$ (here $\left.e_{i}=y_{i}-u_{i}\right)$. Hence

$$
\begin{aligned}
\| A_{1,1}(t) e_{1}(t)+\cdots+A_{1, n}(t) e_{n}(t) \\
\quad+\int_{0}^{t} k_{1,1}(t, s) e_{1}(s) d s \ldots+\int_{0}^{t} k_{1, n}(t, s) e_{n}(s) d s \| \\
\leq\left\|A_{1,1} e_{1}\right\|+\cdots+\left\|A_{1, n} e_{n}\right\| \leftarrow \text { By triangle inequality } \\
\quad+\left\|\int_{0}^{t} k_{1,1}(t, s) e_{1}(s) d s\right\| \ldots+\left\|\int_{0}^{t} k_{1, n}(t, s) e_{n}(s) d s\right\|
\end{aligned}
$$


$\leq\left\|A_{1,1}\right\|\left\|e_{1}\right\|+\cdots+\left\|A_{1, n}\right\|\left\|e_{n}\right\| \leftarrow$ By properties of max norm

$$
+\left\|e_{1}\right\|\left\|\int_{0}^{t}\left|k_{1,1}(t, s)\right| \mathbf{1} d s\right\| \ldots+\left\|e_{n}\right\|\left\|\int_{0}^{t}\left|k_{1, n}(t, s)\right| \mathbf{1} d s\right\|
$$

$\leq M_{1}\left(\left\|e_{1}\right\|+\cdots+\left\|e_{n}\right\|\right) \leftrightarrow$ By continuous conditions

$+M_{2}\left(\left\|e_{1}\right\| \ldots+\left\|e_{n}\right\|\right)$

$\leq \begin{cases}\mathcal{O}\left(h^{m+1-n}\right), & \text { if } \lambda \in[-1,1), \\ \mathcal{O}\left(h^{m+1-2 n}\right), & \text { if } \lambda=1,\end{cases}$

where $M_{1}$ and $M_{2}$ are upper bounds of $\left\|A_{1, j}\right\|$ and $\left\|\int_{0}^{t}\left|k_{1, j}(t, s)\right| \mathbf{1} d s\right\|$ for $j=1, \ldots, n$, respectively and $\mathbf{1}=[1, \ldots, 1]^{T}$. For obtaining (4.21), we also used following inequalities

$$
\begin{aligned}
& \max _{t \in I}\left|\int_{0}^{t} k_{1, j}(t, s) e_{j}(s) d s\right| \\
& \quad \leq \max _{t \in I} \int_{0}^{t}\left|k_{1, j}(t, s) e_{j}(s)\right| d s \leq \max _{t \in I} \int_{0}^{t}\left|k_{1, j}(t, s)\right|\left|e_{j}(s)\right| d s \\
& \quad \leq \max _{t \in I} \int_{0}^{t}\left|k_{1, j}(t, s)\right| \mathbf{1}\left\|e_{j}(s)\right\| d s \leq\left\|e_{j}(s)\right\| \max _{t \in I} \int_{0}^{t}\left|k_{1, j}(t, s)\right| \mathbf{1} d s .
\end{aligned}
$$

The first equation of system (4.20) is a Volterra integral equation of first kind i.e.

$$
\begin{aligned}
\int_{0}^{t} k_{1, n+1}(t, s) y_{n+1}(s) d s= & f_{1}(t)-A_{1,1}(t) y_{1}(t) \ldots-A_{1, n}(t) y_{n}(t) \\
& -\int_{0}^{t} k_{1,1}(t, s) y_{1}(s) d s \ldots-\int_{0}^{t} k_{1, n}(t, s) y_{n}(s) d s .
\end{aligned}
$$

From (4.21), the corresponding discontinuous collocation method for Eq. (4.23) is equivalent to the perturbed discontinuous collocation method with the perturbation term

$$
\delta(h, n, i)= \begin{cases}\mathcal{O}\left(h^{m+1-n}\right), & \text { if } \lambda \in[-1,1), \\ \mathcal{O}\left(h^{m+1-2 n}\right), & \text { if } \lambda=1\end{cases}
$$

Then Theorem 2, yields

$$
\left\|y_{n+1}-u_{n+1}\right\| \leq \begin{cases}\mathcal{O}\left(h^{m-n}\right), & \text { if } \lambda \in[-1,1), \\ \mathcal{O}\left(h^{m-1-2 n}\right), & \text { if } \lambda=1\end{cases}
$$

that completes the proof.

The fully discretised perturbed discontinuous collocation solution $\widehat{u}_{n}\left(t_{n}+\right.$ $\left.s h_{n}\right)=\sum_{j=0}^{m} L_{j}(s) \widehat{U}_{n, j}$, for the fully discretised discontinuous collocation method (FDDCM) is obtained by solving the system

$$
h \sum_{j=1}^{m} a_{i j} k\left(t_{n, i}, t_{n, j}\right) \widehat{U}_{n, j}+h \sum_{l=0}^{n-1} \sum_{j=1}^{m} b_{j} k\left(t_{n, i}, t_{n, j}\right) \widehat{U}_{l, j}=f\left(t_{n, i}\right)+\bar{\delta}(h, n, i) .
$$


The FDDCM has also the same order of convergence which we obtained for the discontinuous collocation method. To prove this, we need a new version of Theorem 2 .

Theorem 4. Let $f(t) \in \mathbf{C}^{m+1}\left(I, R^{r}\right), \frac{\partial^{i} k(t, s)}{\partial t^{i}} \in \mathbf{C}\left(D, R^{r \times r}\right)(i=1, \ldots, m)$, satisfy system (4.2). Also suppose $K(t, t)$ is invertible matrix function for all $t \in I$, and $f(0)=0$. Then the approximate solution $u_{h}$ of the fully discretised perturbed discontinuous collocation method with distinct collocation parameters $c_{1}, \ldots, c_{m} \in(0,1]$, and $c_{m} \leq 1$ (if exists) converges to the solution $y$ for all $m \geq 2$ if and only if $-1 \leq \lambda \leq 1$ and the collocation error satisfies

$$
\left\|y-u_{h}\right\| \leq \begin{cases}\mathcal{O}\left(h^{\min \left\{m_{1}-1, m+1\right\}}\right), & \text { if } \lambda \in[-1,1), \\ \mathcal{O}\left(h^{\min \left\{m_{1}-2, m\right\}}\right), & \text { if } \lambda=1,\end{cases}
$$

where $m_{1}$ is the order of perturbed term.

Proof. Proceeding similarly to the proof of Theorem 2, we show that $\left\|\widehat{u}_{h}-u_{h}\right\|$ has the same order of $\left\|y-u_{h}\right\|$. Then using

$$
\left\|y-\widehat{u}_{h}\right\| \leq\left\|\widehat{u}_{h}-u_{h}\right\|+\left\|y-u_{h}\right\|
$$

completes the proof.

Analyzing the index 2 IAEs of the form (1.2) is a question requested in [5, p. 499], and for this purpose, it is enough to set $\nu=2$, in the previous theorems. This analysis has recently been done (see [11]).

\section{Nonlinear Case}

Suppose that the equation

$$
A(t) y(t)+\int_{0}^{t} \kappa(t, s, y(s)) d s=f(t), \quad t \in I:=[0, T]
$$

has a unique solution. Then, the collocation solution $u_{h} \in\left(\mathcal{S}_{m-1}^{(-1)}\left(I_{h}\right)\right)^{r}$ of the system (5.1) is defined as the solution equation

$$
A(t) u_{h}(t)+\int_{0}^{t} k\left(t, s, u_{h}(s)\right) d s=f(t)
$$

for $t \in X_{h}$. Proceeding as in Section 3, we obtain the following nonlinear equations

$$
A\left(t_{n, i}\right) U_{n, i}+F_{n, i}+h \int_{0}^{c_{i}} \kappa\left(t_{n, i}, t_{n}+s h_{n}, \sum_{j=1}^{m} L_{j}(s) U_{n, j}\right) d s=f\left(t_{n, i}\right)
$$

for $i=1, \ldots, m$, where the lag term is defined by

$$
F_{n, i}=h \sum_{l=0}^{n-1} \int_{0}^{1} \kappa\left(t_{n, i}, t_{l}+s h_{l}, \sum_{j=1}^{m} L_{j}(s) U_{l, j}\right) d s .
$$


A suitable method for solving this system is the Newton's iterative method, since it can be proved (see [1]) that this method converges to the solution $U_{n, i}$ with the initial value $U_{n-1, i}$, for sufficiently small $h$. Subtracting (5.2) from (5.1), we obtain

$$
A(t) e(t)+\int_{0}^{t}\left(\kappa(t, s, y(s))-\kappa\left(t, s, u_{h}(s)\right)\right) d s=0 .
$$

Now suppose $k(t, s, y)$ is continuously differentiable with respect to $y$. Then using the mean value theorem and letting $t=t_{n, i}$, we get

$$
A\left(t_{n, i}\right) e\left(t_{n, i}\right)+\int_{0}^{t_{n, i}} \kappa_{y}\left(t_{n, i}, s, \eta(s)\right) e(s) d s=0,
$$

where $\eta(s)$ is between $y(s)$ and $u(s)$. This is a linear system and its index can be considered as an index definition for the nonlinear system (5.1). Hence, for the nonlinear Hessenberg type system

$$
\begin{aligned}
& {\left[\begin{array}{cccc}
A_{1,1}(t) & \cdots & A_{1, \nu-1}(t) & 0 \\
\vdots & \ddots & \vdots & \vdots \\
A_{\nu-1,1}(t) & \cdots & 0 & 0 \\
0 & \cdots & 0 & 0
\end{array}\right]\left[\begin{array}{c}
y_{1}(t) \\
y_{2}(t) \\
\vdots \\
y_{\nu}(t)
\end{array}\right]} \\
& +\int_{0}^{t}\left[\begin{array}{c}
k_{1}\left(t, s, y_{1}(s), y_{2}(s), \ldots, y_{\nu}(s)\right) \\
\vdots \\
k_{\nu-1}\left(t, s, y_{1}(s), y_{2}(s)\right) \\
k_{\nu}\left(t, s, y_{1}(s)\right)
\end{array}\right] d s=\left[\begin{array}{c}
f_{1}(t) \\
f_{2}(t) \\
\vdots \\
f_{\nu}(t)
\end{array}\right]
\end{aligned}
$$

the index is $\nu$, if $\prod_{i=1}^{\nu} k_{i, y_{\nu+1-i}}\left(t, t, y_{1}(t), \ldots, y_{\nu+1-i}(t)\right)$ is invertible in a neighbourhood of $\left(y_{1}(t), \ldots, y_{\nu}(t)\right)$. We should have the same results for the order of the collocation methods that we obtained in previous sections.

Theorem 5. Let $f(t) \in \mathbf{C}^{m+1}\left(I, R^{r}\right), \frac{\partial^{i} k\left(t, s, y_{1}(s), \ldots, y_{\nu+1-j}(s)\right)}{\partial t^{i}} \in \mathbf{C}\left(D, R^{r \times r}\right)$ for $i=1, \ldots, m$ and $j=1, \ldots, \nu$ and $A(t) \in \mathbf{C}^{m+1}\left(I, R^{r \times r}\right)$ satisfy system (5.4). Suppose that the system has Hessenberg form of index $\nu$. Also suppose that the consistency conditions of Theorem 1 hold for this system. Then the approximate solution $u_{h}$ of the (fully discretised) discontinuous collocation method with distinct collocation parameters $c_{1}, \ldots, c_{m} \in(0,1]$ (if exists) converges to the solution $y$ if and only if

$$
-1 \leq \lambda=(-1)^{m} \prod_{i=1}^{m} \frac{1-c_{i}}{c_{i}} \leq 1 .
$$

Moreover, collocation error satisfies

$$
\left\|y_{i}-u_{i}\right\| \leq \begin{cases}\mathcal{O}\left(h^{m+1-i}\right), & \text { if } \lambda \in[-1,1) \\ \mathcal{O}\left(h^{m+1-2 i}\right), & \text { if } \lambda=1\end{cases}
$$


Table 1. Errors of the discontinuous collocation method for Example 1 with different $N$.

\begin{tabular}{llllll}
\hline$N$ & 16 & 32 & 64 & 128 & 256 \\
\hline$\left\|e_{1}\left(t_{N, 1}\right)\right\|$ & $4.1566 \mathrm{e}-007$ & $5.1406 \mathrm{e}-008$ & $6.3917 \mathrm{e}-009$ & $7.9681 \mathrm{e}-010$ & $9.9455 \mathrm{e}-011$ \\
$\left\|e_{1}\left(t_{N, 2}\right)\right\|$ & $4.9129 \mathrm{e}-008$ & $9.5320 \mathrm{e}-009$ & $1.3936 \mathrm{e}-009$ & $1.8653 \mathrm{e}-010$ & $2.3868 \mathrm{e}-011$ \\
$\left\|e_{1}\left(t_{N, 3}\right)\right\|$ & $3.5239 \mathrm{e}-006$ & $4.2741 \mathrm{e}-007$ & $5.2632 \mathrm{e}-008$ & $6.5299 \mathrm{e}-009$ & $8.1413 \mathrm{e}-010$ \\
$\left\|e_{2}\left(t_{N, 1}\right)\right\|$ & $9.5560 \mathrm{e}-004$ & $2.3208 \mathrm{e}-004$ & $5.7218 \mathrm{e}-005$ & $1.4207 \mathrm{e}-005$ & $3.5376 \mathrm{e}-006$ \\
$\left\|e_{2}\left(t_{N, 2}\right)\right\|$ & $1.1945 \mathrm{e}-004$ & $3.2942 \mathrm{e}-005$ & $8.5943 \mathrm{e}-006$ & $2.1917 \mathrm{e}-006$ & $5.5255 \mathrm{e}-007$ \\
$\left\|e_{2}\left(t_{N, 3}\right)\right\|$ & $5.6018 \mathrm{e}-004$ & $1.4063 \mathrm{e}-004$ & $3.5224 \mathrm{e}-005$ & $8.8139 \mathrm{e}-006$ & $2.2026 \mathrm{e}-006$ \\
$\left\|e_{3}\left(t_{N, 1}\right)\right\|$ & $5.3729 \mathrm{e}-001$ & $2.6906 \mathrm{e}-001$ & $1.3461 \mathrm{e}-001$ & $6.7320 \mathrm{e}-002$ & $3.3626 \mathrm{e}-002$ \\
$\left\|e_{3}\left(t_{N, 2}\right)\right\|$ & $2.0794 \mathrm{e}-001$ & $1.0465 \mathrm{e}-001$ & $5.2481 \mathrm{e}-002$ & $2.6276 \mathrm{e}-002$ & $1.3135 \mathrm{e}-002$ \\
$\left\|e_{3}\left(t_{N, 3}\right)\right\|$ & $4.4270 \mathrm{e}-001$ & $2.1834 \mathrm{e}-001$ & $1.0851 \mathrm{e}-001$ & $5.4095 \mathrm{e}-002$ & $2.6978 \mathrm{e}-002$ \\
\hline
\end{tabular}

for $i=1, \ldots, \nu$. Hence

$$
\left\|y-u_{h}\right\| \leq \begin{cases}\mathcal{O}\left(h^{m+1-\nu}\right), & \text { if } \lambda \in[-1,1), \\ \mathcal{O}\left(h^{m+1-2 \nu}\right), & \text { if } \lambda=1,\end{cases}
$$

where $u_{h}=\left[u_{1}, \ldots, u_{\nu}\right]^{T}$.

\section{$6 \quad$ Numerical Experiments}

The following examples are given to confirm the theoretical results for the system of IAEs of Hessenberg type. The order of the error is slop of function $\log (\|(e(h))\|)$ with respect to $\log (h)$ with $h=1 / N$. To shorten notation we let $b e \pm a$ stands for $b \times 10^{ \pm a}$, in the given tables where $a$ and $b$ are real numbers.

Example 1. Let

$$
A(t)=\left(\begin{array}{lll}
1 & 1 & 0 \\
1 & 0 & 0 \\
0 & 0 & 0
\end{array}\right), \quad k(t, s)=\left(\begin{array}{ccc}
s^{2}+t+1 & 0 & -1 \\
s t+1 & 1 & 0 \\
1 & 0 & 0
\end{array}\right)
$$

and determine $f$ such that the exact solution of (1.1) is $y=\left[e^{t}, \cos (t), e^{t}\right]^{T}$. To solve this index 3 system on $[0,1]$, we use FDDCM with $c=[.1, .6, .9]$. For these values of $c$, we have $\lambda=-0.6667$. Tables 1 and 2 , show the errors and orders of each component at the collocation parameters $t_{N, i}, i=1, \ldots, m$ for different values of $N$. We expect $e_{1}=\mathcal{O}\left(h^{3}\right), e_{2}=\mathcal{O}\left(h^{2}\right)$ and $e_{3}=\mathcal{O}(h)$ which confirm the results of Theorem 3 .

Example 2. Let

$$
\begin{gathered}
A(t)=\left(\begin{array}{ll}
1 & 0 \\
0 & 0
\end{array}\right), \quad k(t, s)=\left(\begin{array}{cc}
1+s t & 1 \\
1 & 0
\end{array}\right), \\
f=\left(\begin{array}{c}
2 \exp (t)+\sin (t)+t(\exp (t)(t-1)+1)-1 \\
\exp (t)-1
\end{array}\right) .
\end{gathered}
$$

Then the exact solution of the index 2 IAE in (1) is given by $y=[\exp (t)$, $\cos (t)]^{T}$. For solving this IAE on $[0,1]$, we use FDDCMs with $c=[.1,0.4, .6, .9]$. For these values of $c$, we have $\lambda=1$. 
Table 2. Order of discontinuous collocation method for Example 1.

\begin{tabular}{llllll}
\hline order of $\backslash N$ & $8-16$ & $16-32$ & $32-64$ & $64-128$ & $128-256$ \\
\hline$\left\|e_{1}\left(t_{N, 1}\right)\right\|$ & 3.0308 & 3.0154 & 3.0077 & 3.0039 & 3.0021 \\
$\left\|e_{1}\left(t_{N, 2}\right)\right\|$ & 0.76612 & 2.3657 & 2.774 & 2.9013 & 2.9663 \\
$\left\|e_{1}\left(t_{N, 3}\right)\right\|$ & 3.0985 & 3.0435 & 3.0216 & 3.0108 & 3.0037 \\
$\left\|e_{2}\left(t_{N, 1}\right)\right\|$ & 2.0899 & 2.0418 & 2.0201 & 2.0099 & 2.0057 \\
$\left\|e_{2}\left(t_{N, 2}\right)\right\|$ & 1.6098 & 1.8585 & 1.9385 & 1.9713 & 1.9879 \\
$\left\|e_{2}\left(t_{N, 3}\right)\right\|$ & 1.9858 & 1.994 & 1.9973 & 1.9987 & 2.0006 \\
$\left\|e_{3}\left(t_{N, 1}\right)\right\|$ & 0.99385 & 0.99781 & 0.99909 & 0.9997 & 1.0015 \\
$\left\|e_{3}\left(t_{N, 2}\right)\right\|$ & 0.97675 & 0.9905 & 0.99576 & 0.99806 & 1.0003 \\
$\left\|e_{3}\left(t_{N, 3}\right)\right\|$ & 1.0501 & 1.0197 & 1.0088 & 1.0043 & 1.0037 \\
\hline
\end{tabular}

Table 3. Errors and order of the discontinuous collocation method for Example 2.

\begin{tabular}{llllll}
\hline$N$ & 8 & 16 & 32 & 64 & 128 \\
\hline$\left\|e_{1}\left(t_{N, 1}\right)\right\|$ & $3.4237 \mathrm{e}-006$ & $4.2369 \mathrm{e}-007$ & $5.2700 \mathrm{e}-008$ & $6.5787 \mathrm{e}-009$ & $7.8815 \mathrm{e}-010$ \\
order & - & 3.0145 & 3.0071 & 3.0019 & 3.0613 \\
$\left\|e_{1}\left(t_{N, 2}\right)\right\|$ & $9.0836 \mathrm{e}-007$ & $1.0912 \mathrm{e}-007$ & $1.3372 \mathrm{e}-008$ & $1.6570 \mathrm{e}-009$ & $1.9780 \mathrm{e}-010$ \\
order & - & 3.0574 & 3.0286 & 3.0126 & 3.0664 \\
$\left\|e_{1}\left(t_{N, 3}\right)\right\|$ & $9.4369 \mathrm{e}-007$ & $1.1126 \mathrm{e}-007$ & $1.3504 \mathrm{e}-008$ & $1.6648 \mathrm{e}-009$ & $1.9798 \mathrm{e}-010$ \\
order & - & 3.0844 & 3.0424 & 3.0200 & 3.0719 \\
$\left\|e_{1}\left(t_{N, 4}\right)\right\|$ & $3.9889 \mathrm{e}-006$ & $4.5793 \mathrm{e}-007$ & $5.4807 \mathrm{e}-008$ & $6.7084 \mathrm{e}-009$ & $7.9538 \mathrm{e}-010$ \\
order & - & 3.1228 & 3.0627 & 3.0303 & 3.0762 \\
$\left\|e_{2}\left(t_{N, 1}\right)\right\|$ & $2.7945 \mathrm{e}-003$ & $1.3852 \mathrm{e}-003$ & $6.8932 \mathrm{e}-004$ & $3.4406 \mathrm{e}-004$ & $1.6942 \mathrm{e}-004$ \\
order & - & 1.0125 & 1.0069 & 1.0025 & 1.0220 \\
$\left\|e_{2}\left(t_{N, 2}\right)\right\|$ & $7.3529 \mathrm{e}-004$ & $3.5580 \mathrm{e}-004$ & $1.7474 \mathrm{e}-004$ & $8.6623 \mathrm{e}-005$ & $4.2503 \mathrm{e}-005$ \\
order & - & 1.0472 & 1.0258 & 1.0124 & 1.0272 \\
$\left\|e_{2}\left(t_{N, 3}\right)\right\|$ & $8.4381 \mathrm{e}-004$ & $3.8165 \mathrm{e}-004$ & $1.8105 \mathrm{e}-004$ & $8.8182 \mathrm{e}-005$ & $4.2874 \mathrm{e}-005$ \\
order & - & 1.1447 & 1.0759 & 1.0378 & 1.0404 \\
$\left\|e_{2}\left(t_{N, 4}\right)\right\|$ & $3.5730 \mathrm{e}-003$ & $1.5708 \mathrm{e}-003$ & $7.3461 \mathrm{e}-004$ & $3.5526 \mathrm{e}-004$ & $1.7209 \mathrm{e}-004$ \\
order & - & 1.1856 & 1.0965 & 1.0481 & 1.0457 \\
\hline
\end{tabular}

Table 3 shows the errors and orders of each component at the collocation parameters $t_{N, i}, i=1, \ldots, m$ for different values of $N$. This table shows that the order of the errors cannot be exceeded from what we proved in the case $\lambda=1$. Hence this order is optimal. This table confirms the phenomenon of decreasing order of collocation methods by two, when the index of the method increases by one for the special case $\lambda=1$.

Finally we give the following example for the nonlinear IAEs.

Example 3. Let

$$
\begin{aligned}
& A(t)=\left(\begin{array}{lll}
1 & 1 & 0 \\
1 & 0 & 0 \\
0 & 0 & 0
\end{array}\right), \quad f=\left(\begin{array}{c}
t+\cos (t)+e^{t}+e^{t}(t-1) \\
t+e^{t}+t^{3} / 3-1 \\
e^{t}-1
\end{array}\right), \\
& k_{1}\left(t, s, y_{1}, y_{2}, y_{3}\right)=y_{1} y_{2}+y_{3}, \quad k_{2}\left(t, s, y_{1}, y_{2}\right)=y_{1}^{2}+y_{2}, \quad k_{3}\left(t, s, y_{1}\right)=e^{y_{1}}
\end{aligned}
$$

with the exact solution $y=\left[t, e^{t}, \sin (t)\right]^{T}$. For this system $\frac{\partial k_{1}}{\partial y_{3}} \frac{\partial k_{2}}{\partial y_{2}} \frac{\partial k_{3}}{\partial y_{1}}=e^{y_{1}}$, which is positive for all $y_{1}$. Hence the index of this nonlinear system on $I:=$ $[0,1]$, is 3 . 
Table 4. Errors of the discontinuous collocation method for Example 3 with different $N$.

\begin{tabular}{llllll}
\hline$N$ & 16 & 32 & 64 & 128 & 256 \\
\hline$\left\|e_{1}\left(t_{N, 1}\right)\right\|$ & $4.1307 \mathrm{e}-007$ & $5.1246 \mathrm{e}-008$ & $6.3817 \mathrm{e}-009$ & $7.9619 \mathrm{e}-010$ & $9.9416 \mathrm{e}-011$ \\
$\left\|e_{1}\left(t_{N, 2}\right)\right\|$ & $4.4454 \mathrm{e}-008$ & $9.0671 \mathrm{e}-009$ & $1.3592 \mathrm{e}-009$ & $1.8422 \mathrm{e}-010$ & $2.3719 \mathrm{e}-011$ \\
$\left\|e_{1}\left(t_{N, 3}\right)\right\|$ & $2.1431 \mathrm{e}-006$ & $2.7125 \mathrm{e}-007$ & $3.4118 \mathrm{e}-008$ & $4.2780 \mathrm{e}-009$ & $5.3535 \mathrm{e}-010$ \\
$\left\|e_{2}\left(t_{N, 1}\right)\right\|$ & $8.3787 \mathrm{e}-004$ & $2.1734 \mathrm{e}-004$ & $5.5372 \mathrm{e}-005$ & $1.3976 \mathrm{e}-005$ & $3.5087 \mathrm{e}-006$ \\
$\left\|e_{2}\left(t_{N, 2}\right)\right\|$ & $1.2327 \mathrm{e}-004$ & $3.3216 \mathrm{e}-005$ & $8.6160 \mathrm{e}-006$ & $2.1937 \mathrm{e}-006$ & $5.5275 \mathrm{e}-007$ \\
$\left\|e_{2}\left(t_{N, 3}\right)\right\|$ & $5.3283 \mathrm{e}-004$ & $1.3711 \mathrm{e}-004$ & $3.4778 \mathrm{e}-005$ & $8.7577 \mathrm{e}-006$ & $2.1956 \mathrm{e}-006$ \\
$\left\|e_{3}\left(t_{N, 1}\right)\right\|$ & $4.8332 \mathrm{e}-001$ & $2.5491 \mathrm{e}-001$ & $1.3099 \mathrm{e}-001$ & $6.6404 \mathrm{e}-002$ & $3.3396 \mathrm{e}-002$ \\
$\left\|e_{3}\left(t_{N, 2}\right)\right\|$ & $1.9202 \mathrm{e}-001$ & $1.0047 \mathrm{e}-001$ & $5.1410 \mathrm{e}-002$ & $2.6005 \mathrm{e}-002$ & $1.3067 \mathrm{e}-002$ \\
$\left\|e_{3}\left(t_{N, 3}\right)\right\|$ & $3.9526 \mathrm{e}-001$ & $2.0497 \mathrm{e}-001$ & $1.0487 \mathrm{e}-001$ & $5.3178 \mathrm{e}-002$ & $2.6749 \mathrm{e}-002$ \\
\hline
\end{tabular}

Table 5. Order of discontinuous collocation method for Example 3.

\begin{tabular}{llllll}
\hline order of $\backslash N$ & $8-16$ & $16-32$ & $32-64$ & $64-128$ & $128-256$ \\
\hline$\left\|e_{1}\left(t_{N, 1}\right)\right\|$ & 3.0218 & 3.0109 & 3.0054 & 3.0028 & 3.0016 \\
$\left\|e_{1}\left(t_{N, 2}\right)\right\|$ & 0.6219 & 2.2936 & 2.7379 & 2.8833 & 2.9573 \\
$\left\|e_{1}\left(t_{N, 3}\right)\right\|$ & 2.9642 & 2.9820 & 2.9910 & 2.9955 & 2.9984 \\
$\left\|e_{2}\left(t_{N, 1}\right)\right\|$ & 1.9249 & 1.9468 & 1.9727 & 1.9863 & 1.9939 \\
$\left\|e_{2}\left(t_{N, 2}\right)\right\|$ & 1.7761 & 1.8919 & 1.9468 & 1.9737 & 1.9887 \\
$\left\|e_{2}\left(t_{N, 3}\right)\right\|$ & 1.9171 & 1.9583 & 1.9791 & 1.9896 & 1.9960 \\
$\left\|e_{3}\left(t_{N, 1}\right)\right\|$ & 0.8535 & 0.9230 & 0.9605 & 0.9801 & 0.9916 \\
$\left\|e_{3}\left(t_{N, 2}\right)\right\|$ & 0.8732 & 0.9345 & 0.9667 & 0.9833 & 0.9929 \\
$\left\|e_{3}\left(t_{N, 3}\right)\right\|$ & 0.8990 & 0.9474 & 0.9668 & 0.9797 & 0.9913 \\
\hline
\end{tabular}

For solving this IAE on $I$, we use FDDCMs with the same $c$ that we used for Example 1.

Tables 4 and 5, show the errors and orders of each component at the collocation parameters $t_{N, i}, i=1, \ldots, m$ for different values of $N$.

\section{Strongly Equivalent}

Consider the IAE

$$
\widetilde{A}(t) \widetilde{y}(t)+\int_{0}^{t} \widetilde{k}(t, s, \widetilde{y}(s)) d s=\widehat{f}(t), \quad t \in I:=[0, T],
$$

such that $\widetilde{A}(t)=E A(t) F(t), \widetilde{k}(t, s, y(s))=E k(t, s, F(s) y(s))$ and $\widehat{f}(t)=$ $E f(t)$, where $E \in R^{r \times r}$ is a nonsingular matrix and $F \in \mathbf{C}\left(I, R^{r \times r}\right)$ is a pointwise nonsingular matrix function. Then we say, systems (5.1) and (7.1) are strongly equivalent. Let $y$ and $\widetilde{y}$ be a solutions of (5.1) and (7.1), respectively. Then it is straightforward to verify that the relation $\widetilde{y}(t)=F^{-1}(t) y(t)$ is a one-to-one correspondence between the corresponding solution sets. Let $u_{h}$ and $\widetilde{u}_{h}$ also be the corresponding numerical solutions of applying FDCCMs to (1.1) and its strongly equivalent form, respectively. Then one can easily prove that $\widetilde{u}_{h}\left(t_{n, i}\right)=F^{-1}\left(\left(t_{n, i}\right)\right) u_{h}\left(t_{n, i}\right)$. This fact generalize the obtained order results of Hessenberg type IAEs to IAEs which are strongly equivalent with Hessenberg type. 


\section{Conclusion}

In this paper, we investigated the integral algebraic equations of Hessenberg type. We also stated a useful existence and uniqueness theorem for IAEs and we analyzed the convergence properties of discontinuous collocation methods and perturbed discontinuous collocation methods for IAEs and SVIEs, respectively. The analysis showed that the order of collocation methods decreases by one when the index of method increases by one for the case $\lambda<1$ and it decreases two times when the stability parameters $\lambda$ is equal to one. From the numerical examples we guess that each IAE of index $\nu$ has the same convergence properties that we obtained for Hessenberg type, which is an open problem.

\section{Acknowledgment}

This is part of the author's Ph. D. thesis, written under the supervision of Dr. S. Shahmorad and Dr. G. Hojjati, at the University of Tabriz.

\section{References}

[1] K. Atkinson. Theoretical Numerical Analysis, a Functional Analysis Framework. Springer, 2001.

[2] K.E. Brenan, S.L. Campbell and L.R. Petzold. Numerical Solution of Initial Value Problems in Differential-Algebraic Equations. North-Holland, Amsterdam, 1989.

[3] H. Brunner. Discretization of Volterra integral equations of the first kind. Math. Comput., 31(139):708-716, 1977. http://dx.doi.org/10.1090/S0025-5718-1977-0451794-6.

[4] H. Brunner. Discretization of Volterra integral equations of the first kind (II). Numer. Math., 30(2):117-136, 1978. http://dx.doi.org/10.1007/BF02042940.

[5] H. Brunner. Collocation Methods for Volterra Integral and Related Functional Equations. Cambridge University Press, 2004.

[6] M.V. Bulatov. Transformations of differential-algebraic systems of equations. Zhurnal Vychislitel'noi Matematiki i Matematicheskoi Fiziki, 34(3):360-372, 1994.

[7] M.V. Bulatov. Regularization of degenerate integro-differential equations. Comput. Math. Math. Phys., 42(11):1602-1608, 2002.

[8] V.F. Chistyakov. Algebro-Differential Operators with Finite-Dimensional Core. Nauka, Siberian Publishing Company RAS, Novosibirsk, 1996.

[9] V.F. Chistyakov. Differential-algebraic equations with a small nonlinear term. Differential Equations, 45(9):1365-1368, 2009.

[10] C.W. Gear. Differential algebraic equations, indices and integral algebraic equations. SIAM J. Numer. Anal., 27(6):1527-1534, 1990.

http://dx.doi.org/10.1137/0727089.

[11] F. Ghoreishi, M. Hadizadeh and S. Pishbin. On the convergence analysis of the spline collocation method for system of integral algebraic equations of index- 2 . Int. J. Comput. Methods, 9(4):131-148, 2012. http://dx.doi.org/10.1142/S021987621250048X. 
[12] M. Hadizadeh, F. Ghoreishi and S. Pishbin. Jacobi spectral solution for integral algebraic equations of index-2. Appl. Numer. Math., 61(1):131-148, 2011. http://dx.doi.org/10.1016/j.apnum.2010.08.009.

[13] E. Hairer, Ch. Lubich and M. Roch. The Numerical Solution of DifferentialAlgebraic Systems by Runge-Kutta Methods, volume 1409 of Lecture Notes in Mathematics. Springer, Berlin, 1989.

[14] F.R.D. Hoog and R. Weiss. High order methods for Volterra integral equations of the first kind. SIAM J. Numer. Anal., 10(4):647-664, 1973. http://dx.doi.org/10.1137/0710057.

[15] F.R.D. Hoog and R. Weiss. On the solution of Volterra integral equations of the first kind. Numer. Math., 21(1):22-32, 1973. http://dx.doi.org/10.1007/BF01436183.

[16] J.-P. Kauthen. The numerical solution of integral-algebraic equations of index 1 by polynomial spline collocation methods. Math. Comp., 70(236):1503-1514, 2001. http://dx.doi.org/10.1090/S0025-5718-00-01257-6.

[17] J.-P. Kauthen and H. Brunner. Continuous collocation approximations to solutions of first kind volterra equations. Math. Comp., 66(220):1441-1459, 1997. http://dx.doi.org/10.1090/S0025-5718-97-00905-8.

[18] P. Kunkel and Mehrmann. Differential-algebraic equations: analysis and numerical solution. European Mathematical Society, 2006.

[19] R. Marz. Numerical Methods for Differential Algebraic Equations. HumboldtUniv., Fachbereich Mathematik, Informationsstelle, 1991.

[20] B. Shiri, S. Shahmorad and G. Hojjati. Convergence analysis of piecewise continuous collocation methods for higher index integral algebraic equations of Hessenberg type. AMCS, 23(2):341-355, 2013. 